\title{
Uusiorersily
}

\section{Using Eye-Tracking Technology to Capture the Visual Attention of Nurses During Interpretation of Patient Monitoring Scenarios from a Computer Simulated Bedside Monitor}

Currie, J., Bond, R. R., McCullagh, P. J., Black, P., Finlay, D., \& Peace, A. (2016). Using Eye-Tracking

Technology to Capture the Visual Attention of Nurses During Interpretation of Patient Monitoring Scenarios from a Computer Simulated Bedside Monitor. In Unknown Host Publication Institute of Sound and Communications Engineers. http://uir.ulster.ac.uk/36088/3/currie_isce.docx

Link to publication record in Ulster University Research Portal

Published in:

Unknown Host Publication

Publication Status:

Published (in print/issue): 13/04/2016

\section{Document Version}

Author Accepted version

\section{General rights}

Copyright for the publications made accessible via Ulster University's Research Portal is retained by the author(s) and / or other copyright owners and it is a condition of accessing these publications that users recognise and abide by the legal requirements associated with these rights.

\section{Take down policy}

The Research Portal is Ulster University's institutional repository that provides access to Ulster's research outputs. Every effort has been made to ensure that content in the Research Portal does not infringe any person's rights, or applicable UK laws. If you discover content in the Research Portal that you believe breaches copyright or violates any law, please contact pure-support@ulster.ac.uk. 


\title{
Using Eye-Tracking Technology to Capture the Visual Attention of Nurses During Interpretation of Patient Monitoring Scenarios from a Computer Simulated Bedside Monitor
}

\author{
${ }^{1}$ Jonathan Currie, ${ }^{1}$ Raymond R. Bond, ${ }^{1}$ Paul McCullagh, ${ }^{2}$ Pauline Black, ${ }^{3}$ Dewar D. Finlay, ${ }^{4}$ Aaron \\ Peace
${ }^{1}$ School of Computing \& Mathematics, Shore Road, Ulster University, Newtownabbey, N. Ireland, United Kingdom ${ }^{2}$ School of Nursing, Northland Road, Ulster University, Londonderry, N. Ireland, United Kingdom ${ }^{3}$ School of Engineering, Shore Road, Ulster University, Newtownabbey, N. Ireland, United Kingdom ${ }^{4}$ Altnagelvin Area Hospital, Glenshane Road, Londonderry, N. Ireland, United Kingdom

\section{Introduction:}

This study analysed the utility of eye tracking technology for gaining insight into the decision making processes of nurses during their interpretation of patient scenarios and vital signs.

\section{Methods:}

Five patient monitoring scenarios (vignette, vital signs [ECG, BP etc.] and scoring criteria) were designed and validated by critical care experts. Participants were asked to interpret these scenarios whilst 'thinking aloud'. Visual attention was measured using infrared lightbased eye-tracking technology. Each interpretation was scored out of 10 . Subjects comprised of students $(n=36)$ and qualified nurses $(n=11)$. Scores and self-rated confidence (where 1=low, 10=high) are presented using mean \pm SD. Significance testing was performed using a $t$-test and ANOVA where appropriate $(\alpha=0.05)$. Multivariate regression was performed to determine if a machine could use eye gaze features to accurately predict competency (dependent variable=score). Independent eye gaze only variables were used in the regression models if they statistically significantly $(p<0.05)$ correlated with the score.

\section{Results:}

Scores across all scenarios were calculated (students $=4.58 \pm 1.13$ vs. qualified $=6.85 \pm 0.82$ ) with statistical significance between groups $(p=<0.01)$. Mean self-rated confidence was also calculated (students $=5.79 \pm 1.05$ vs. qualified $=7.49 \pm 1.00, p=<0.01$ ). There was a weak positive correlation between confidence and score amongst students $(r=0.323, p=0.06)$, although no meaningful correlation with qualified nurses $(r=-0.099, p=0.77)$. However, for all participants there was a moderate correlation between confidence and score $(r=0.592$, $\mathrm{p}=<0.01$ ).

The fitness of the regression models for predicting competency based on eye gaze features only is as follows:

- Scenario 1: $R^{2}=0.407$, Std Error $=1.243(p=0.09)$

- Scenario 2: $R^{2}=0.746$, Std Error $=1.439(p=0.01)$

- Scenario 3: $R^{2}=0.385$, Std Error $=1.564(p=0.03)$

- Scenario 4: $R^{2}=0.687$, Std Error $=1.340(p=0.44)$

- Scenario 5: $R^{2}=0.766$, Std Error $=0.960(p=0.02)$

The following table also shows where subjects fixated the most and least on the different vital signs on the bedside monitor (note the lower fixation duration on the ECG by students in comparison to qualified nurses). 
Table 1 - Total fixation duration on the different areas on screen.

\begin{tabular}{|c|c|c|c|c|}
\hline Group & $\begin{array}{l}\text { Order of Highest } \\
\text { Fixation Duration }\end{array}$ & Areas on Screen & Mean \% & SD (seconds) \\
\hline \multirow{6}{*}{ Qualified Nurses } & 1 & ECG & $30 \%$ & 25.37 \\
\hline & 2 & Arterial Blood Pressure & $28 \%$ & 22.71 \\
\hline & 3 & Oxygen Saturation & $22 \%$ & 18.65 \\
\hline & 4 & Central Venous Pressure & $9 \%$ & 7.22 \\
\hline & 5 & Temperature & $6 \%$ & 5.38 \\
\hline & 6 & Respiratory Rate & $5 \%$ & 4.95 \\
\hline & & & & \\
\hline \multirow[t]{6}{*}{ Student Nurses } & 1 & Arterial Blood Pressure & $33 \%$ & 18.95 \\
\hline & 2 & Oxygen Saturation & $20 \%$ & 12.52 \\
\hline & 3 & ECG & $17 \%$ & 13.54 \\
\hline & 4 & Central Venous Pressure & $13 \%$ & 11.49 \\
\hline & 5 & Temperature & $9 \%$ & 4.97 \\
\hline & 6 & Respiratory Rate & $8 \%$ & 6.43 \\
\hline \multicolumn{3}{|c|}{ Area on Screen } & \multicolumn{2}{|c|}{ t-test } \\
\hline \multicolumn{3}{|c|}{ ECG } & \multicolumn{2}{|c|}{0.07} \\
\hline \multicolumn{3}{|c|}{ Arterial Blood Pressure } & \multicolumn{2}{|c|}{0.50} \\
\hline \multicolumn{3}{|c|}{ Oxygen Saturation } & \multicolumn{2}{|c|}{0.77} \\
\hline \multicolumn{3}{|c|}{ Central Venous Pressure } & \multicolumn{2}{|c|}{0.07} \\
\hline \multicolumn{3}{|c|}{ Temperature } & \multicolumn{2}{|c|}{0.08} \\
\hline \multicolumn{3}{|c|}{ Respiratory Rate } & \multicolumn{2}{|c|}{0.13} \\
\hline
\end{tabular}

\section{Conclusion:}

The study has shown that eye-tracking measurements can provide insight into the decisionmaking of nurses and can be used to predict competency. 